University of Wollongong

Research Online

Faculty of Engineering and Information

Faculty of Engineering and Information

Sciences - Papers: Part A

Sciences

$1-1-2015$

\title{
Microgrid capability diagram: a tool for optimal grid-tied operation
}

Athmi Jayawardena

University of Wollongong, avj998@uowmail.edu.au

Lasantha Meegahapola

University of Wollongong, lasantha.meegahapola@rmit.edu.au

Duane A. Robinson

University of Wollongong, duane@uow.edu.au

Sarath Perera

University of Wollongong, sarath@uow.edu.au

Follow this and additional works at: https://ro.uow.edu.au/eispapers

Part of the Engineering Commons, and the Science and Technology Studies Commons

Research Online is the open access institutional repository for the University of Wollongong. For further information contact the UOW Library: research-pubs@uow.edu.au 


\title{
Microgrid capability diagram: a tool for optimal grid-tied operation
}

\begin{abstract}
The microgrid paradigm has gained much interest in the electricity industry due to the increased penetration of distributed resources. A grid-tied microgrid can be viewed as a single generating entity or a load depending on its power export and import at the grid supply point. Similar to conventional generators, grid-tied microgrids have the potential to be able to participate in the energy market in the future to achieve technical, financial and environmental benefits. Effective participation in the energy markets requires numerous planning tools and a comprehensive understanding of the full capability of the microgrid. This paper presents a systematic approach for developing a capability diagram for a gridtied microgrid which represents the active and reactive power exchange capability of the microgrid with the main grid. Capability diagrams have been developed for two different microgrids and the impacts of different modelling aspects and network conditions have been analysed using several case studies. Effects of plug-in hybrid electric vehicles, capacitor banks, and other storage devices on microgrid capability diagram have also been addressed in this paper. Furthermore, operating points of the capability diagram have been verified using time domain simulations.
\end{abstract}

Keywords

tied, operation, grid, capability, optimal, microgrid, tool, diagram

Disciplines

Engineering | Science and Technology Studies

\section{Publication Details}

A. V. Jayawardena, L. G. Meegahapola, D. A. Robinson \& S. Perera, "Microgrid capability diagram: a tool for optimal grid-tied operation," Renewable Energy, vol. 74, pp. 497-504, 2015.

This journal article is available at Research Online: https://ro.uow.edu.au/eispapers/3002 


\title{
Microgrid Capability Diagram: A Tool for Optimal \\ Grid-tied Operation
}

A.V. Jayawardena ${ }^{a}$, L. G. Meegahapola ${ }^{a,}{ }^{*}$ D. A. Robinson ${ }^{a}$, S. Perera ${ }^{a}$

${ }^{a}$ Australian Power Quality and Reliability Centre, School of Electrical, Computer and Telecommunications Engineering, University of Wollongong, Wollongong, New South Wales, 2522, Australia

\begin{abstract}
The microgrid paradigm has gained much interest in the electricity industry due to the increased penetration of distributed resources. A grid-tied microgrid can be viewed as a single generating entity or a load depending on its power export and import at the grid supply point. Similar to conventional generators, grid-tied microgrids have the potential to be able to participate in the energy market in the future to achieve technical, financial and environmental benefits. Effective participation in the energy markets requires numerous planning tools and a comprehensive understanding of the full capability of the microgrid. This paper presents a systematic approach for developing a capability diagram for a grid-tied microgrid which represents the active and reactive power exchange capability of the microgrid with the main grid. Capability diagrams have been developed for two different microgrids and the impacts of different modelling aspects and network conditions have been analysed using several case studies. Effects of plug-in hybrid electric vehicles, capacitor banks, and other storage devices on microgrid capability diagram have also been addressed in this paper. Furthermore, operating points of the capability diagram have been verified using time domain simulations.
\end{abstract}

Keywords: Capability diagram, distributed generation, distribution system, grid-tied microgrid, optimisation

\section{Introduction}

Increasing penetration of localised energy generation (PV systems, wind generators, microturbines etc.), and storage devices (batteries, flywheels etc.) at distribution level has paved the way for the microgrid paradigm to become a reality. Integration of multiple microgrids into the utility grid will allow microgrids to provide ancillary services to the utility during normal operation and to provide emergency services to adjacent microgrids during a main grid outage. Besides technical and financial benefits, due to being an enabler of renewable and high efficiency distributed generation resources, operation of microgrids can also provide environmental benefits by reducing the overall carbon footprint and creating zero-net-energy communities [1]. Grid-tied microgrids can behave as single generating units or as loads in a technically and economically feasible way.

\footnotetext{
${ }^{*}$ Coorresponding author. Tel +61242213408 Fax +61242213236

Email addresses: avj998@uowmail.edu.au (A.V. Jayawardena), lasantha@ieee.org (L. G. Meegahapola), duane@uow.edu.au (D. A. Robinson), sarath@uow.edu.au (S. Perera)
} 
Similar to the large generators in traditional power systems, microgrids could participate in wholesale markets to supply energy and other ancillary services to the network in the future [2].

Few research activities have been carried out on developing microgrid central control systems for a multi-microgrid environment. In [3], optimal resource allocation within the microgrid is carried out by a microgrid central controller (MCC) and it has been assumed that there is no limit to the power exchanged with the main grid. Participation of microgrids in the open market has been considered in [4] which enable microgrids to trade power with the main grid. However, in [3] and [4], the distribution network service provider (DNSP) or the distribution management system is unable to obtain the overall active and reactive power capabilities of the microgrid within the technical constraints for the considered time interval. Most of the research in this area has been focused on optimising the power availability and minimising operation costs within the distribution network while minimising power flow through the grid supply point (GSP) [5-8]. Allowing grid-tied microgrids to participate in energy and ancillary service markets while fulfilling the local energy demand will provide opportunity for additional revenue for the microgrids, however, considerable planning and understanding of the technical capabilities of the microgrid as an energy resource is required. In order to enable this operation and to make decisions in the market, MCCs and DNSPs must not only find the optimum operating conditions, but they must also be aware of the full capability of the microgrid at the GSP.

Capability diagrams are considered essential system planning tools and are widely used in power system operation. The concept of the capability diagram is traditionally related to synchronous machines, and represents the area of permissible operation in terms of active and reactive power available at the machine terminal $[9,10]$. Recent research has been carried out on developing capability diagrams for wind turbine based doubly-fed-induction-generator (DFIG) [11, 12], grid connected PV units [13], generator-transformer units [14], and for HVDC links [15]. A graphical method to determine the network limits of a wind farm is presented in [16]. For a multiple node system, the capability diagram is obtained by reducing the network into a two-node system and using Thevenin's equivalent for the system at the particular node of interest. However, practicability of this method reduces with the increasing size of the network.

The total load supplying capability of a power system is modelled and represented as a capability diagram in [17] using an optimisation approach subjected to technical and operating constraints. In [18], reactive power support from networks comprising only voltage controlling wind generators has been analysed, where a P-Q capability diagram has been derived without taking the local loads into account. Deriving a diagram for the maximum power transfer capability of a grid-tied microgrid is much different from individual distributed generators (DGs) or wind farms due to the complexity of the microgrid, caused by the network configuration, network constraints and the combination of different DGs and loads. 
This paper introduces a new approach of developing an active and reactive power capability diagram corresponding to the GSP of a microgrid, while considering microgrid generation capabilities, local load demand and network constraints. Various features such as different load modelling aspects, individual machine limitations, and effects of plug-in hybrid electric vehicles (PHEVs) and reactive power devices are considered in developing the capability diagram. Capability diagrams are derived based on an optimisation model in MATLAB for two different microgrid models, which are validated using DIgSILENT PowerFactory. This graphical representation of the active and reactive power capability at the GSP can be utilised as a new tool for grid-tied microgrids in market operation. This tool is required to assist in understanding the full capability of a microgrid, to allow optimum use of distributed energy resource (DERs), and to provide coordinated support to the network through ancillary services as required.

Structure of the paper is as follows; Section 2 presents the optimisation model and development of a simplified capability diagram for an example microgrid. Derivation of a more detailed and realistic capability diagram with various case studies are presented in Section 3. Impacts of the operation of PHEVs, storage devices, and capacitor banks on microgrid capability are presented in Section 4. Discussion and conclusions are given in Section 5 and Section 6 respectively.

\section{Development of Microgrid Capability Diagram}

\subsection{Non-linear optimisation model}

In this section, the development of a capability diagram is demonstrated for a grid-tied microgrid. The capability diagram is derived using the optimisation technique. Objective function for the optimisation model is developed so as to maximise the active power flow through the GSP while minimising the active power losses in the microgrid.

Objective function;

$$
\operatorname{Max}\left(P_{G D P}-\sum P_{\text {loss }}\right)
$$

where $P_{G S P}$ is the active power through the grid supply point and $\sum P_{l o s s}$ is the total active power loss in the microgrid.

The objective function is subjected to the general load flow equalities expressed in (2) and (3).

$$
\begin{array}{r}
P_{g i}-P_{l i}-P_{i}(V, \delta)=0 \\
Q_{g i}-Q_{l i}-Q_{i}(V, \delta)=0
\end{array}
$$

where $P_{g i}, Q_{g i}$ are the active and reactive power outputs from the generator at node $i, P_{l i}, Q_{l i}$ are the active and reactive power demands of the load at node $i$, and $P_{i}, Q_{i}$ are the active and reactive power flow through node $i$. 


$$
\begin{aligned}
& P_{i}(V, \delta)=V_{i} \sum V_{k} Y_{i k} \cos \left(\delta_{i}-\delta_{k}-\emptyset_{i k}\right) \\
& Q_{i}(V, \delta)=V_{i} \sum V_{k} Y_{i k} \sin \left(\delta_{i}-\delta_{k}-\emptyset_{i k}\right)
\end{aligned}
$$

where $\emptyset_{i k}$ is the phase angle of the line admittance between node $i$ and node $k, \delta_{i}$ is the phase angle of the voltage at nodes $i$ with respect to the reference node, $V_{i}$ is the magnitude of the voltage at node $i$, and $Y_{i k}$ is the magnitude of the line admittance between node $i$ and node $k$.

The capability diagram is derived while maintaining all the network voltages and line currents within the rated values. Inequalities which will account for the limits on magnitude and phase angle of nodal voltages are given by (6) and (7);

$$
\begin{gathered}
V_{i}^{\text {min }} \leq V_{i} \leq V_{i}^{\text {max }} \\
-\pi \leq \delta_{i} \leq \pi
\end{gathered}
$$

Distribution line capacity limit is given by;

$$
I_{i k}(V, \delta) \leq I^{\max }
$$

where $I_{i k}$ is the magnitude of the current flow from node $i$ to node $k$;

$$
I_{i k}(V, \delta)=\frac{\sqrt{V_{i}^{2}+V_{k}^{2}-2 V_{i} V_{k} \cos \left(\delta_{i}-\delta_{k}\right)}}{Z_{i k}}
$$

where $Z_{i k}$ is the magnitude of the line impedance between node $i$ and node $k$.

Power flow between the microgrid and the main grid is limited by the rating of the coupling transformer at the GSP. Thus, the transformer power limit is incorporated using (10);

$$
\sqrt{P_{t r}^{2}+Q_{t r}^{2}} \leq S_{t r}
$$

where $S_{t r}$ is the rated apparent power of the transformer.

Furthermore, the maximum and minimum generator power capability limits are incorporated in to the optimisation model by;

$$
\begin{aligned}
Q_{g i}^{\min } \leq Q_{g i} & \leq Q_{g i}^{\max } \\
P_{g i}^{\min } \leq P_{g i} & \leq P_{g i}^{\max }
\end{aligned}
$$

Reactive power through the GSP is increased iteratively using (3), and the optimisation model is solved at each step to obtain the maximum active power flow through the GSP. This procedure is carried out for both power import and export modes in order to develop the complete capability diagram while the objective function is subjected to typical AC power flow constraints expressed in 
(2) - (12). Numerical solutions for the non-linear optimisation problem are obtained by using the interior-point algorithm (fmincon) [19] available in the optimisation solver within MATLAB.

\subsection{Microgrid model-1}

An example microgrid, model-1, comprising of a 5 MVA synchronous generator (SG) based hydro power plant and a 1.5 MVA DFIG based wind power plant is illustrated in Fig. 1. The microgrid is connected to the utility through a 10 MVA, $69 \mathrm{kV} / 13.8 \mathrm{kV}$ transformer. Load 1 and Load 2 are considered as an industrial customer (4.8 MW peak, 0.85 p.f. lagging) and a commercial customer (1.5 MW peak, 0.85 p.f. lagging) respectively. During this study, the tap position of the coupling transformer is assumed to be fixed.

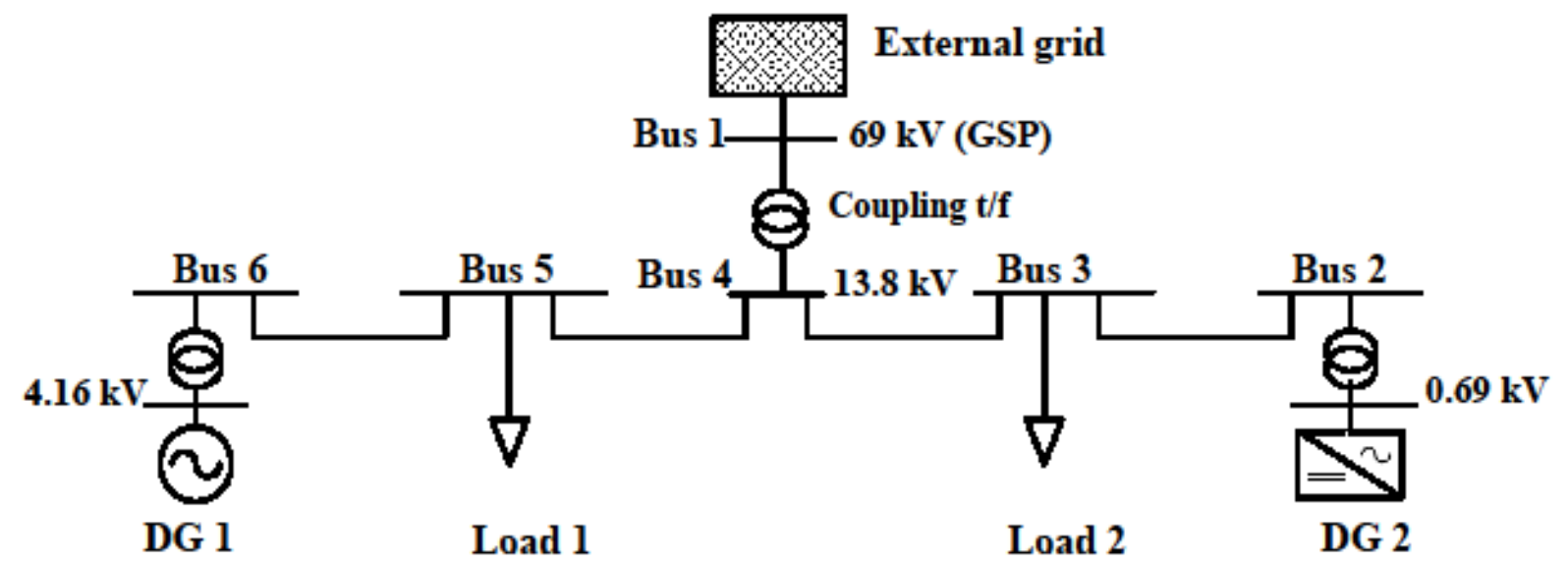

Figure 1: Single line diagram of the microgrid model-1

\subsection{A simplified capability diagram}

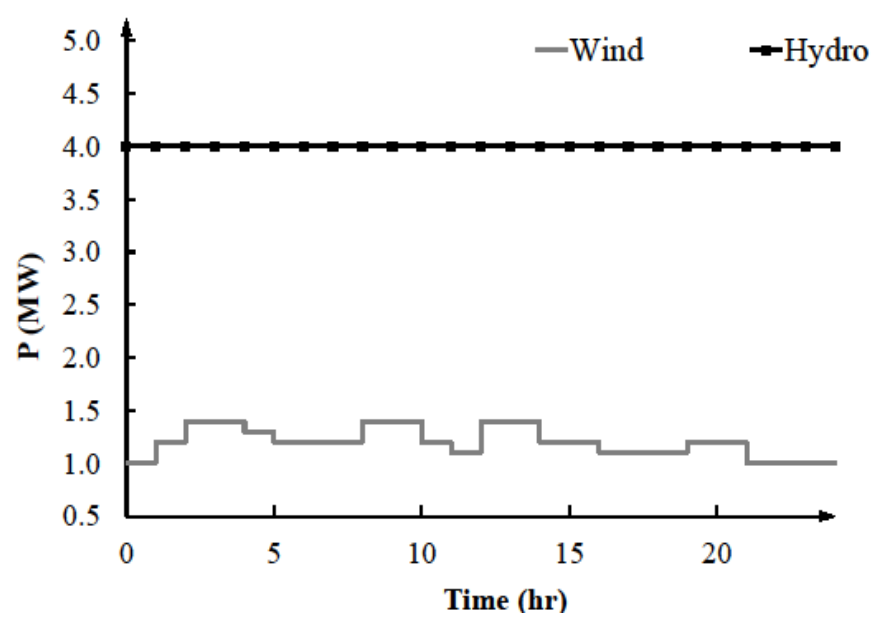

(a) 


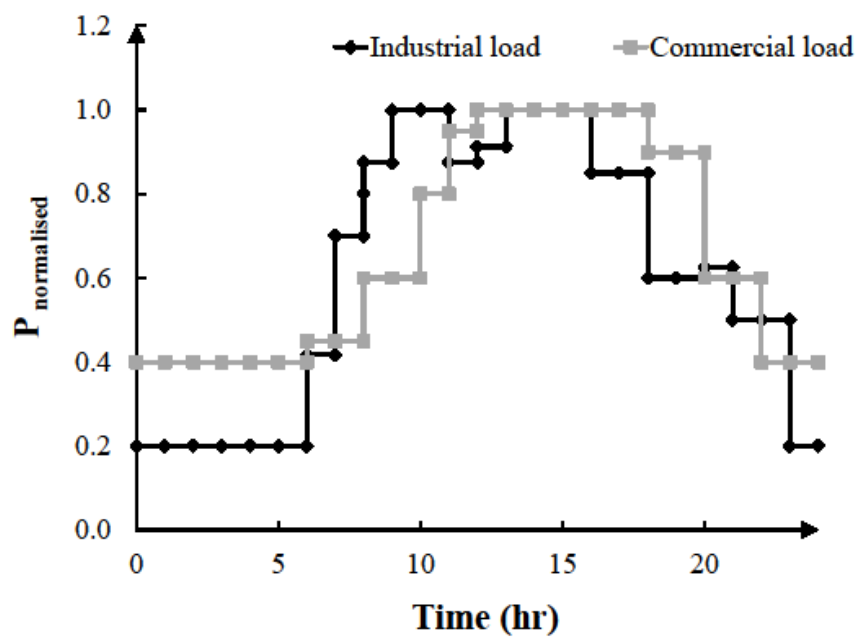

(b)

Figure 2: (a) Daily generation profiles of the SG and the DFIG, and (b) Daily load demand profiles for Load-1 and Load-2 during a typical weekday in summer

Daily generation and demand profiles on a typical weekday in summer, as illustrated in Fig. 2 (a) and Fig.2 (b) [20] are considered for the derivation of microgrid capability diagram. Due to the intermittent nature of wind and time varying load demand patterns, the capability diagram of the microgrid also varies with time. For the purpose of analysis, in this section, the capability diagram is derived for the period when the microgrid has minimum load (1.56 MW) and maximum generation (5.4 MW). Voltage at the GSP is maintained at $1 \angle 0^{\circ} \mathrm{pu}$ and voltage regulation (VR) within the microgrid is taken as $\pm 5 \%$. Loads are modelled as constant power loads such that $P_{l i}$ and $Q_{l i}$ have fixed values independent of the voltage. These constraint parameters would generally need to be adjusted to suit the particular network requirements. Values for the line capacities, transformer ratings and generator ratings as expressed in (8) - (12) are also incorporated into the optimisation model.

Reactive power through the GSP is increased iteratively using (3), and the optimisation model is executed at each step to obtain the maximum active power flow through the GSP. This procedure is carried out for both power import and export modes in order to develop the complete capability diagram. Fig. 3 illustrates the simplified capability diagram corresponding to the microgrid model-1, where positive values indicate power export from the microgrid and negative values represent power import into the microgrid. It can be observed that during the particular time period, the microgrid is capable of exporting a maximum of 3.8 MW of active power and 3.2 MVAr of reactive power. Furthermore, the microgrid demonstrates the capability of operating in all four modes of power import/export combinations. 


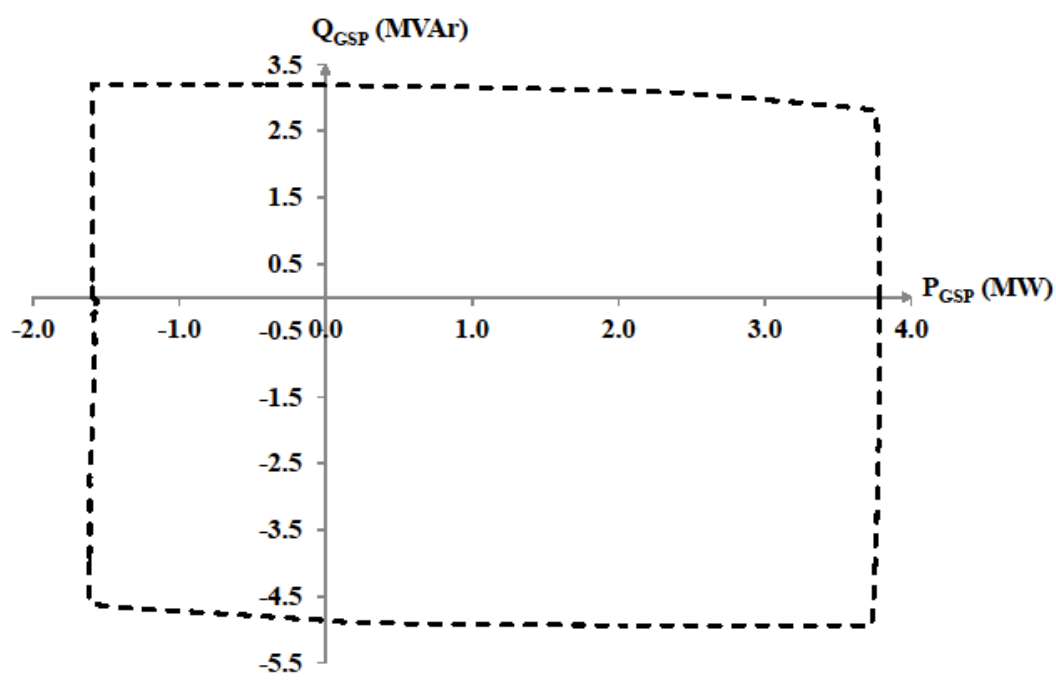

Figure 3: Simplified capability diagram for the microgrid model-1 for the period with minimum load and maximum generation

\section{Detailed Capability Diagram and Case Studies}

The capability diagram methodology developed in Section 2.1 is further improved in this section by incorporating individual capability limits of the DGs and modelling voltage dependency of loads. Furthermore, case studies are carried out to analyse the impacts of DG outages, voltage regulation, and variations in local loading conditions on microgrid power exchange capability. For this purpose, a more complex and realistic microgrid model is developed based on the modified IEEE 13-Bus system [21] comprising of two DFIGs (each 1.5 MVA) and a SG (5 MVA). The microgrid model-2 illustrated in Fig. 4 is connected to the main grid through a 10 MVA, 115 kV/4.16 kV transformer. Generation profiles used in Section 2 are repeated in this study and details of the load models are presented in Table 2. In order to support the network voltage, a fixed capacitor bank rated at 0.5 MVAr is connected to node-634. Daily load profiles of an industrial load feeder, a commercial load feeder and a residential load feeder are illustrated in Fig. 2 (b) and Fig. 5 respectively.

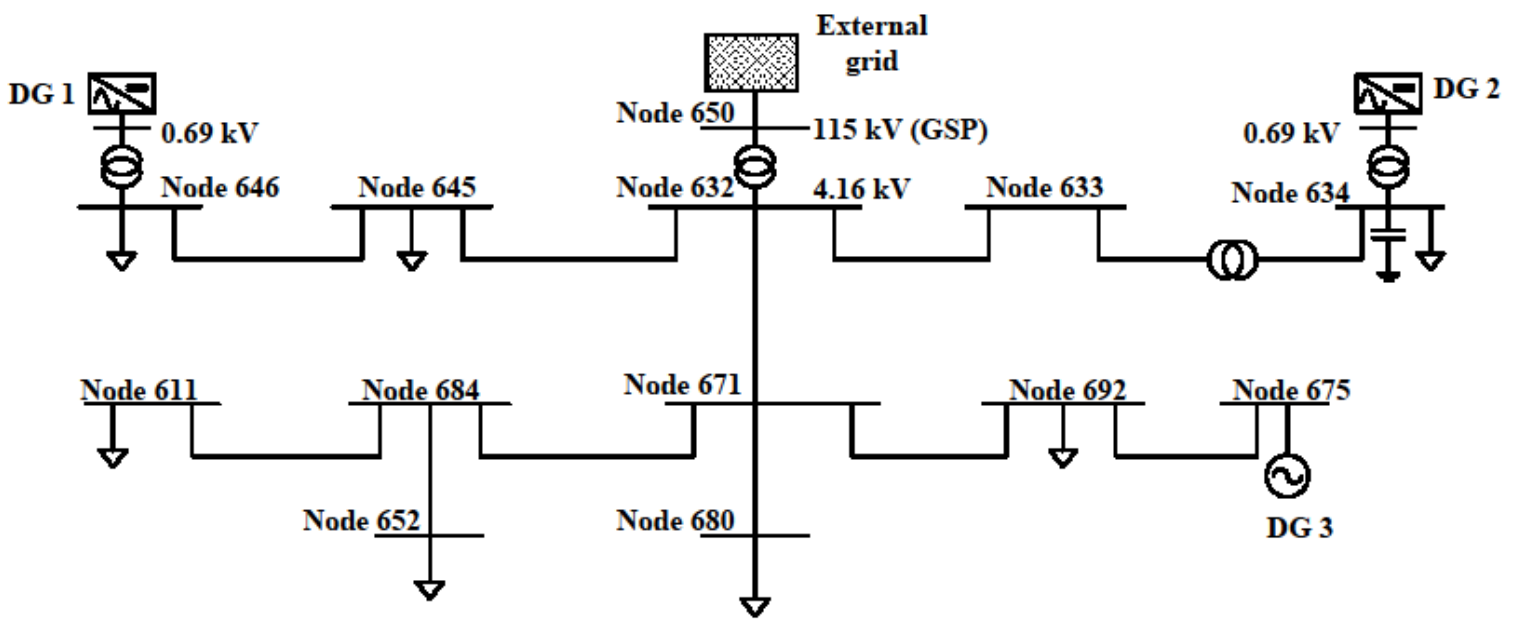

Figure 4: Simplified capability diagram for the microgrid model-2 
Table 2: Details of loads in microgrid model-2 [22]

\begin{tabular}{cccccc}
\hline Load-bus & Load class & $P_{o}(M W)$ & p.f & $\alpha$ & B \\
\hline \hline 611 & Residential & 0.3 & 0.90 & 1.2 & 2.9 \\
\hline 634 & Residential & 0.6 & 0.90 & 1.2 & 2.9 \\
\hline 645 & Commercial & 0.5 & 0.85 & 0.99 & 3.5 \\
\hline 646 & Industrial & 0.5 & 0.85 & 0.18 & 6.0 \\
\hline 652 & Industrial & 0.2 & 0.85 & 0.18 & 6.0 \\
\hline 680 & Commercial & 0.5 & 0.85 & 0.99 & 3.5 \\
\hline 692 & Industrial & 0.8 & 0.85 & 0.18 & 6.0 \\
\hline
\end{tabular}

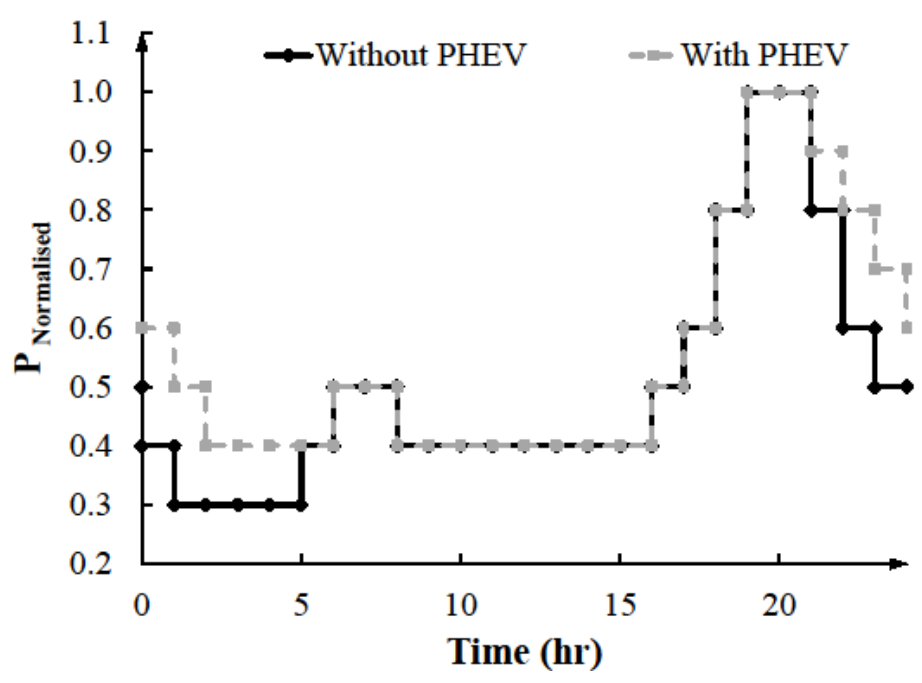

Figure 5: Daily load profiles for a residential feeder with and without PHEV during a typical weekday in summer

\subsection{Impact of individual DG capability limits}

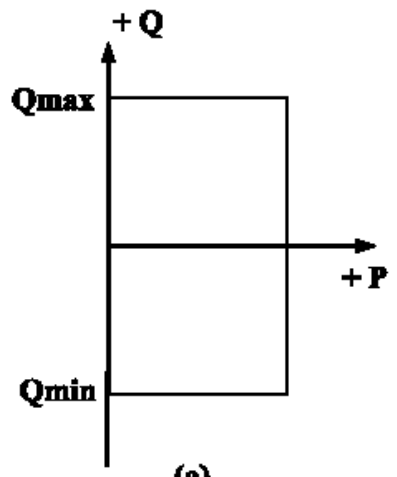

(a)

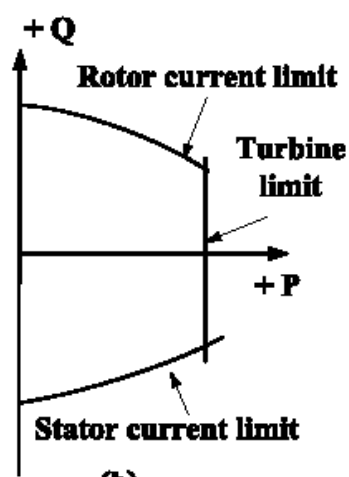

(b)

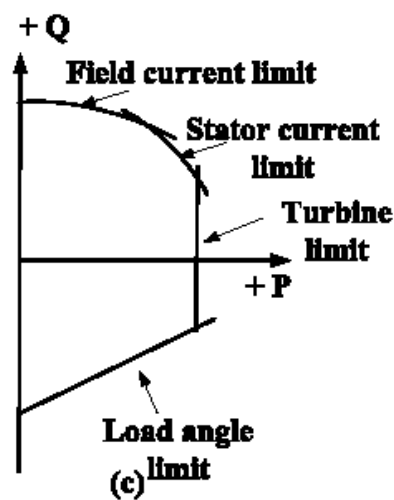

(c) ${ }^{\text {limit }}$

Figure 6: Capability diagram of (a) a generator neglecting the machine limits (b) a DFIG, and (c) a SG

This section presents the impact of neglecting the individual machine limitations on the microgrid capability diagram. As illustrated in Fig. 6 (b), the power capability of a DFIG is mainly determined by the rotor current limit, stator current limit and the wind turbine rating [11, 12]. Similarly, the power output capability of a SG based hydro power plant is limited by the stator current limit, rotor current limit, load angle and the hydro-turbine rating [22] as illustrated in Fig. 6 (c), while 
disregarding those machine limitations would result in a simplified rectangular DG capability diagram as in Fig. 6 (a).

In the optimisation model, (1) - (10) are kept unchanged as in Section 2.1. First, the optimisation model is solved with constant values as the maximum and minimum power limits in (11) and (12). For the next scenario, both (11) and (12) are changed accordingly by incorporating the machine limitations as illustrated in Fig. 6 (b) and Fig. 6 (c). Fig. 7 illustrates the two capability diagrams developed for the generation and load demand conditions at 1.00 p.m. (day peak) with constant power load models.

As expected, it can be observed that ignoring the real machine capability limits would result in a higher capability compared to the actual microgrid capability. In the particular capability diagram in Fig. 7, when individual machine limitations are ignored, microgrid capability diagram indicates a maximum active power export of $3.6 \mathrm{MW}$, which is $52 \%$ greater than what is actually available from the microgrid. Therefore, it is essential to incorporate accurate capability limits of individual DGs for optimisation model.

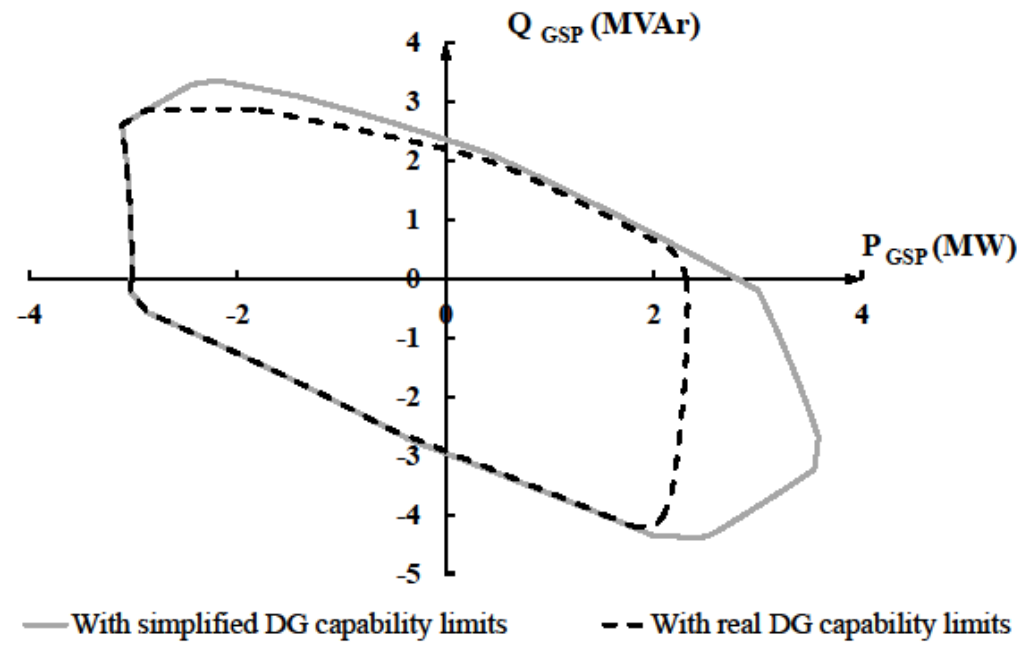

Figure 7: Impact of simplistic and real DG capability limits on the capability diagram of microgrid model-2

\subsection{Impact of voltage dependency of loads}

Active and reactive power demands of loads vary with the load bus voltage. Hence, this section analyses the impact of voltage dependency of loads on microgrid capability diagram by modelling the loads in (2) and (3) as follows;

$$
P_{l i}=P_{o i}\left(\frac{V_{i}}{V_{o i}}\right)^{\alpha} \quad Q_{l i}=Q_{o i}\left(\frac{V_{i}}{V_{o i}}\right)^{\beta}
$$

where $P_{o i}, Q_{o i}$ are active and reactive power at voltage $V_{o i}$ respectively. In the optimisation model, equations remain the same as explained in Section 2.1 with generator limits incorporated as explained in Section 3.1. The corresponding factors for the loads in the microgrid model-2 are given in Table 2. 
Capability diagrams for the microgrid model-2 are derived during the day peak (1.00 p.m.) and are illustrated in Fig. 8. It can be observed that the reactive power export capability of the microgrid has been affected by the voltage dependency of loads. Reactive power exponent of the load models considered in this scenario are relatively larger than the active power exponent, which affects the reactive power limits of the capability diagram compared to the active power limits.

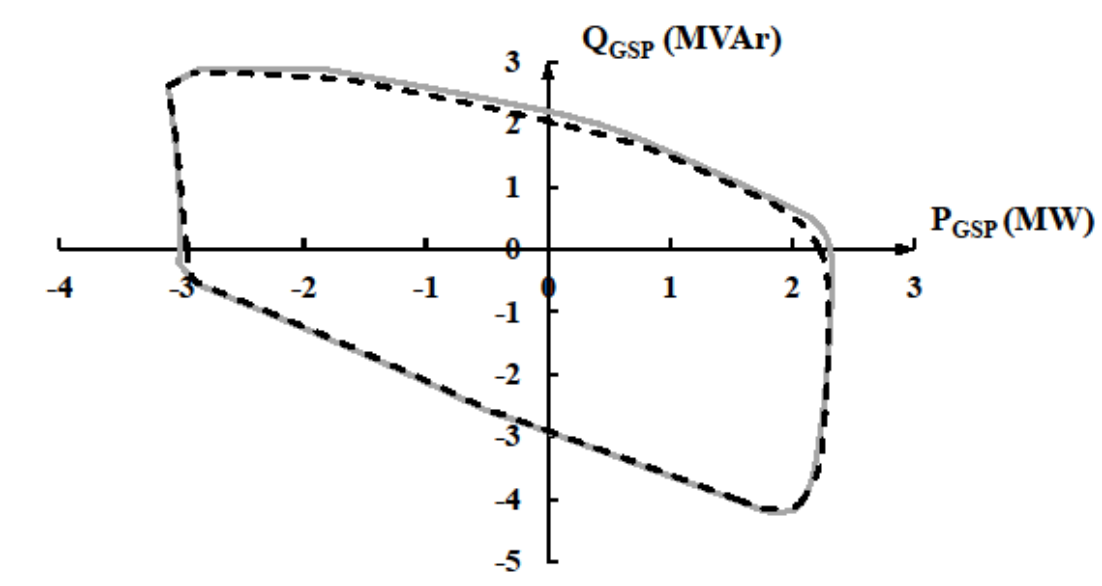

-With constant power load models

- - With voltage dependent load models

Figure 8: Impact of voltage dependent load modelling on the capability diagram of microgrid model-2

\subsection{Variations of capability diagram with different load demand conditions}

Impact of the time varying generation and load demand patterns on the power exchange capability of the microgrid is analysed in this scenario. In the optimisation model, equations remain the same as in Section 2.1 with generator limits incorporated as explained in Section 3.1, and loads modelled as in Section 3.2. Capability diagrams are derived at different times of the day such that the variation of generation and load demands are captured. Capability diagrams for the microgrid model-2 at 3.00 a.m. (minimum load and maximum generation), at 1.00 p.m. (maximum load and maximum generation) and at 8.00 p.m. with different load and generation conditions are illustrated in Fig. 9.

As expected, microgrid has a higher capability to export power to the upstream network when the difference between the local load demand and the local power generation is high. As an example, with same power generation in the microgrid model-2, capability diagram at $3.00 \mathrm{a}$.m. with minimum load has shifted more into the positive active power area (right hand side of the P-Q plane) compared to the capability diagram at 1.00 p.m. with maximum loading. Comparing the capability diagrams derived for the two microgrid models in Section 2 and Section 3, it is evident that the shape and size of the capability diagram varies with microgrid generation and loading conditions, as different constraints/limits will be reached in the optimisation model at each load flow level. 


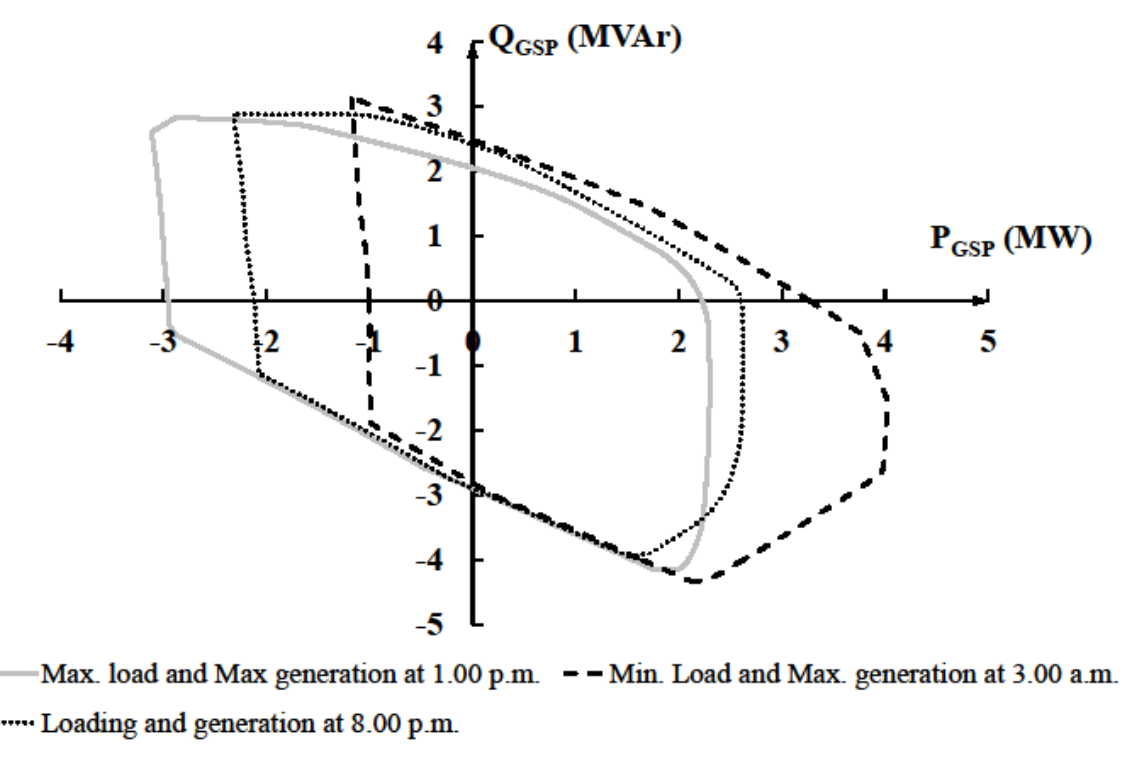

Figure 9: Variations of capability diagram with different loading conditions in microgrid model-2

\subsection{Impact of DG outage on capability diagram}

Fig. 10 illustrates the impacts of individual DG outages on the power exchange capability between the microgrid model-2 and the external grid under peak loading conditions (1.00 p.m.). Capability diagrams are obtained by disconnecting a single generator at a time, while maintaining the same local load demand. For this scenario, equations corresponding to generator limits and the generator transformer in the optimisation model are removed. It can be observed that irrespective of the location, outages of DG 1 and DG 2 (identical DGs) have much similar impact on the microgrid power export capability. Furthermore, an outage of the largest DG (DG 3) will require the particular microgrid to import active power from the external grid. Thus, this type of a graphical tool will be useful for the microgrid controllers to establish an overall idea about the microgrid capability and to optimally operate the remaining DGs during a major generator outage.

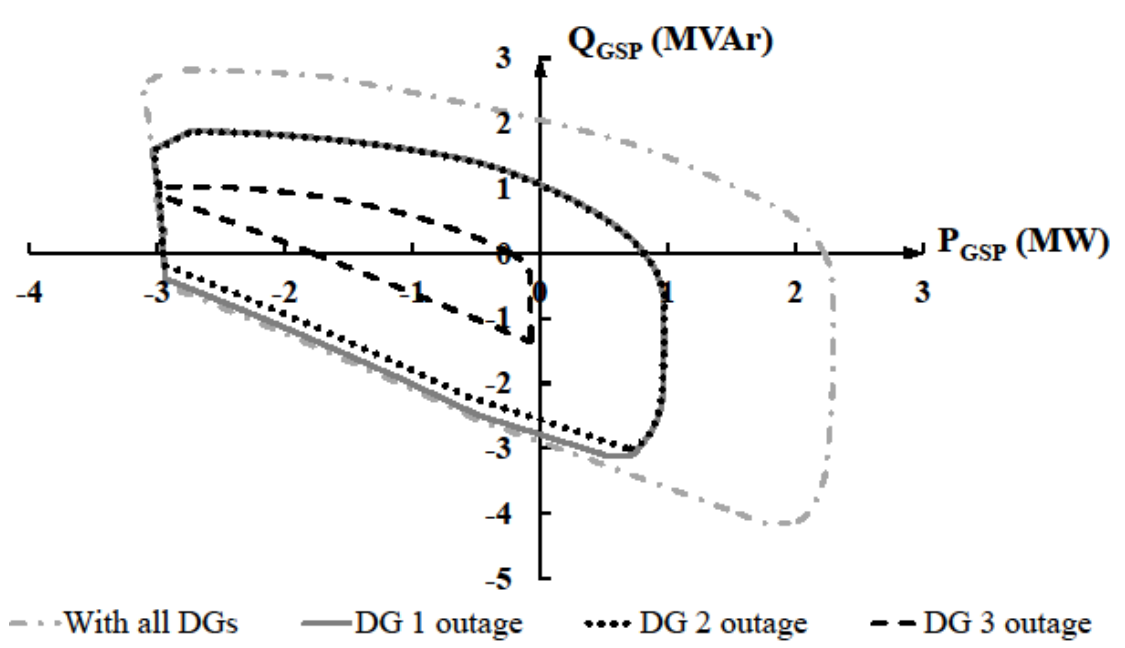

Figure 10: Impacts of individual DG outages on the capability diagram of microgrid model-2 


\subsection{Impact of voltage regulation on microgrid capability limits}

This section presents the impacts of different voltage regulation levels of the microgrid buses and the GSP on the capability limits. For different voltage regulations, limits in (6) are changed accordingly and the optimisation model is executed as described in Section 2.1. As expected, it can be observed from Fig. 11 that an increase in the range of acceptable voltage variation at the buses within the microgrid is favourable for power export from the microgrid (capability diagrams (b) and (c)). Furthermore, for same local loading conditions, power import and export capability of the microgrid can be increased by allowing the voltage at the GSP to be varied within an acceptable limit, rather than fixing at a certain voltage level (capability diagrams (a) and (b)). In energy market operation, the MCC or the DNSP can refer the microgrid capability diagram and increase the microgrid capability by issuing suitable control commands to the bus voltage regulators.

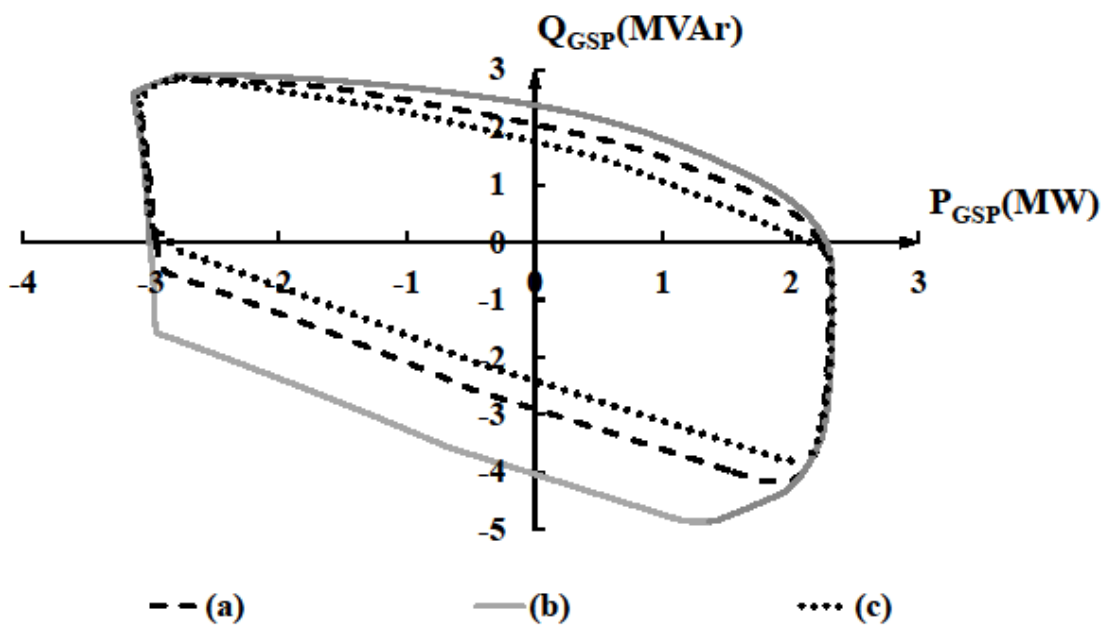

Figure 11: Impacts of voltage regulation on the capability diagram of microgrid model-2 with (a) $V_{G S P}=1 \mathrm{pu}$ and microgrid $\pm 5 \%$, (b) $V_{G S P}= \pm 2 \%$ and microgrid $V R \pm 5 \%$, (c) $V_{G S P}=1$ and microgrid $V R \pm 2 \%$

\section{Impacts of PHEVs, Capacitor Banks and Other Storage Devices on the Capability Diagram}

PHEVs are becoming popular due to their low emission and efficient mode of transportation compared to fossil fuel based vehicles [23]. They are connected to the distribution network with other loads for charging and discharging purposes. In the first part of this section, the impact of charging and discharging of PHEVs on microgrid capability diagram is analysed. For this study, it is assumed that the residential feeders at node-634 and node-611 have load profiles as illustrated in Fig. 5 which include the aggregated effect of PHEV charging, where charging occurs mainly during the off-peak hours due to dual tariff [23]. Capability diagrams are obtained as described in Section 2.1. Fig. 12 compares the capability diagrams at 3.00 a.m. with and without PHEV charging at the residential feeders. During the charging process, PHEV could consume power from the local resources available 
within the microgrid or import power from the external grid. Charging of PHEV will reduce the power export capability of the microgrid.

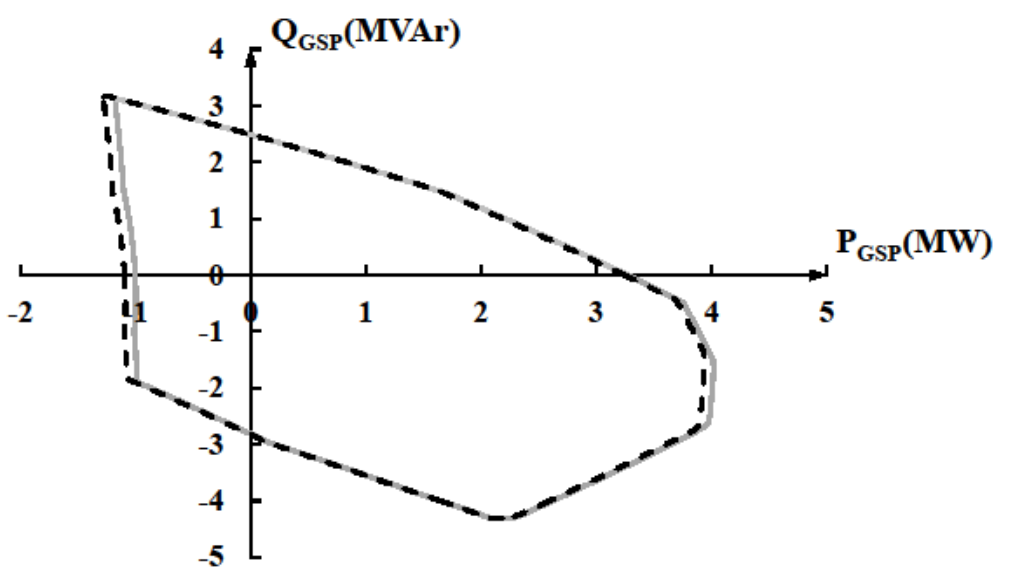

-Without PHEV loads _ - With PHEV loads at Nodes-634 and Node-611

Figure 12: Capability diagrams of the microgrid model-2 at 3.00 a.m. with and without PHEV charging at residential loads

Apart from residential charging, EV charging stations can be constructed in commercial and industrial feeders [24]. In the next scenario, it is assumed that PHEV charging stations are located in two commercial feeders (node-645 and node-680) and two industrial feeders (node-652 and node692) with a morning peak of 0.1 MW at each station occurring at 9.00 a.m., when PHEV owners charge their vehicles after coming to work or to the city. Fig. 13 presents the capability diagrams with fleets of PHEV charging at different stations. It can be observed that regardless of the location of the charging stations, the aggregated effect of small scale fleets of charging PHEVs can be represented in the microgrid power import and export capability diagram.

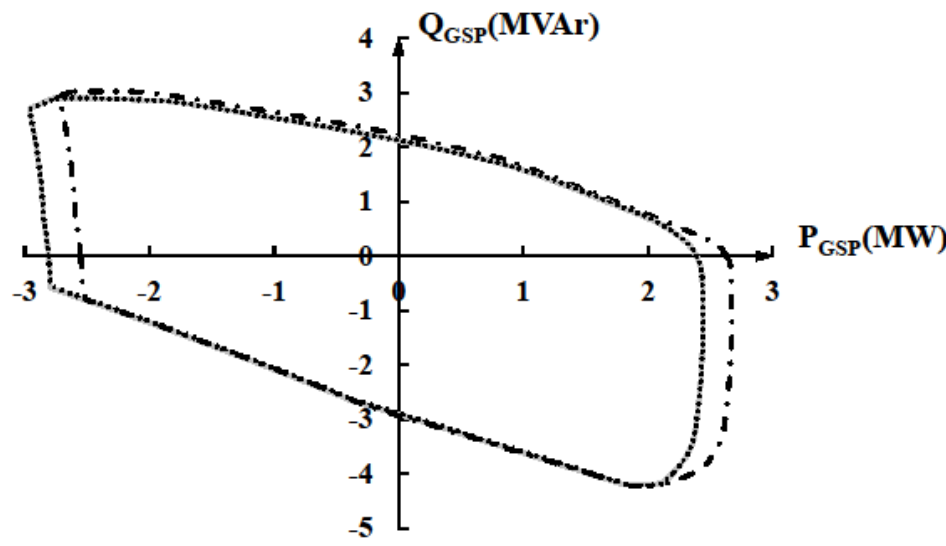

- - Without PHEV loads

- PHEV loads at Nodes 692, 652, and 645

...... PHEV loads at Nodes 692, 652, and 680

Figure 13: Capability diagrams for the microgrid model-2 at 9.00 a.m. with PHEV charging stations 
In the next scenario, Vehicle-to-Grid (V2G) concept is considered where fleets of PHEVs discharge in order to supply active power back to the grid for peak shaving [25]. Fleets of PHEVs are assumed to supply 0.1 MW of power for nearly one hour from stations located at node-645 and node680 during the day-peak hour. It can be observed from Fig. 14 that V2G can be used to cater the local demand which increases the microgrid power export capability. Storage devices within the microgrid can be utilised to reduce the power import from the external grid. In general, storage devices are utilised to mitigate power mismatch, to smooth out variability in renewable energy generations, to reduce network losses, and to obtain financial benefits in the energy market. When deriving the power import/export capability diagram for a grid-tied microgrid, storage devices can be modelled as loads or generators depending on the operation. Energy stored in an individual PHEV is negligible relative to that of the grid. However, fleets of vehicles with considerable number of PHEVs can be aggregated in order to be considered as a feasible storage option. The aggregator who is controlling the power exchange between the PHEVs and the grid will receive requests for different ancillary services from the microgrid operator and will issue commands to fleets of PHEVs to operate in the V2G mode [26].

Typically, capacitor banks are used to support the local voltage by injecting reactive power. In the energy market paradigm, capacitor banks can contribute to export reactive power from the microgrid to the external grid. However, such operation requires careful analysis of financial and technical aspects prior to being incorporated in to the microgrid capability diagram.

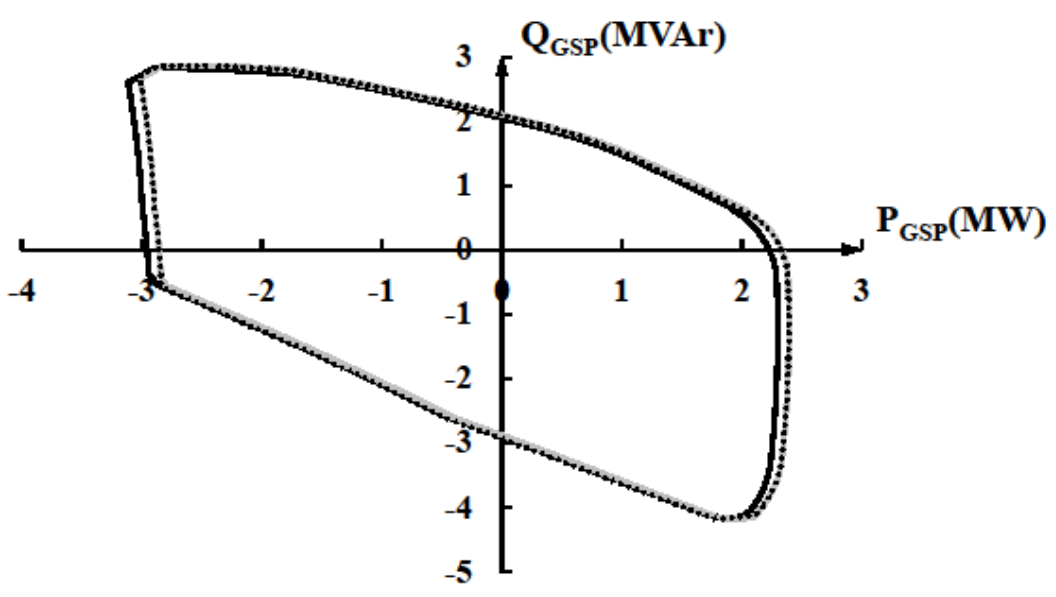

-Without V2G —With V2G atNode $645 \quad$ W.... With V2G at Node 680

Figure 14: Capability diagrams for the microgrid model-2 during day-peak with V2G application

\section{Discussion}

Due to time varying load and generation patterns, capability diagram of a microgrid will vary with time and it is not practical for the MCC to derive capability diagrams for an entire day. However, MCC can derive the capability diagrams for pre-defined time periods or by using the forecasted 
generation and demand patterns at critical operation times of the microgrid (i.e. day-peak, night-peak, minimum load, and maximum generation). Furthermore, the capability diagram can be derived online provided proper communication media exists between the generators, load centres and the MCC.

These capability diagrams can be utilised by the MCC or the DNSP for bidding in the energy market in the future. The proposed methodology and the mathematical model have the flexibility to incorporate any optimisation algorithm and any constraint related to the microgrid for developing capability diagrams. DNSPs and MCCs have the opportunity to run optimisation algorithms to optimise different objective functions subjected to different financial and technical constraints. Those optimum operating points are located within the microgrid capability diagram. Through a capability diagram, the maximum possible power can be harnessed from a grid-tied microgrid. Furthermore, in a multi-microgrid environment where microgrids trade between themselves, capability diagrams of individual microgrids can be used as a tool in deciding the maximum amount of power exchange.

\section{Conclusions}

This paper presented a new systematic approach for developing capability diagrams for gridtied microgrids. An optimisation model was developed under various network constraints and the solutions were plotted on a complex power plane to derive the capability limits for two different microgrid models. Impacts of individual DG capability limits, load modelling, voltage regulation, DG outages, and PHEV operation on the microgrid capability limits were analysed. Furthermore, practical aspects of the implementation of microgrid capability diagram were discussed. Depending on the requirement of the DNSP or the MCC, capability diagram of a grid-tied microgrid can be utilised as a graphical tool to gain a better understanding of the microgrid capability, to allow optimum use of DERs and to provide ancillary services support to the external network.

\section{References}

[1] F. Farzan, S. Lahiri, M. Kleinberg, K. Gharieh, F. Farzan, M. Jafari, Microgrids for fun and profit: The economics of installation investments and operations, IEEE Power and Energy Magazine, 11 (2013) 52-58.

[2] M. Smith, D. Ton, Key Connections: The U.S. Department of Energy's microgrid initiative, IEEE Power and Energy Magazine, 11 (2013) 22-27.

[3] A. Dimeas, N. Hatziargyriou, Operation of a multiagent system for microgrid control, IEEE Trans. Power Syst., 20 (2005) 1447-1455.

[4] A. Tsikalakis, N. Hatziargyriou, Centralized control for optimizing microgrids operation, IEEE Trans. Energy Convers., 23 (2008) 241-248.

[5] S. Arefifar, Y. Mohamed, T. H. M. EL-Fouly, Supply-adequacy-based optimal construction of microgrids in smart distribution systems, IEEE Trans. Smart Grid, 3 (2012) 1491-1502. 
[6] A. Gabash, P. Li, Active-reactive optimal power flow in distribution networks with embedded generation and battery storage, IEEE Trans. Power Syst., 27 (2012) 2026-2035.

[7] L. Ochoa, A. Keane, G. Harrison, Minimizing the reactive support for distributed generation: enhanced passive operation and smart distribution networks, IEEE Trans. Power Syst., 26 (2011) 2134-2142.

[8] S. Paudyal, C. Canizares, K. Bhattacharya, Optimal operation of distribution feeders in smart grids, IEEE Trans. Ind. Electron., 58 (2011) 4495-4503.

[9] IEEE guide for operation and maintenance of turbine generators, IEEE Std. 67-2005 (Revision of IEEE Std 67-1990), 2006, 1-69.

[10] N. E. Nilsson, J. Mercurio, Synchronous generator capability curve testing and evaluation, IEEE Trans. Power Del., 9 (1994) 414-424.

[11] S. Engelhardt, I. Erlich, C. Feltes, J. Kretschmann, F. Shewarega, Reactive power capability of wind turbines based on doubly fed induction generators, IEEE Trans. Energy Convers., 26 (2011) 364-372.

[12] T. Lund, P. E. Sørensen, J. Eek, Reactive power capability of a wind turbine with doubly fed induction generator, Wind Energy, John/Wiley \& Sons Ltd., 10 (2007) 379-39.

[13] F. Delfino, G. Denegri, M. Invernizzi, R. Procopio, G. Ronda, A P-Q capability chart approach to characterize grid connected pv-units integration of wide-scale renewable resources into the power delivery system, CIGRE/IEEE PES Joint Symposium, (2009).

[14] A. Losi, M. Russo, P. Verde, D. Menniti, Capability chart for generator-transformer units, 8th Mediterranean Electrotechnical Conference, 3 (1996) 1580-1583.

[15] J. De Silva, C. Arnold, J. Arrillaga, Capability chart for an HVDC link, IEE Gen. Transm. Distrib., 134 (1987) 181-186.

[16] S. Abdelkader, D. Flynn, Graphical determination of network limits for wind power integration, IET Gen. Transm. Distrib., 3 (2009) 841-849..

[17] E. Chiodo, A. Losi, R. Mongelluzzo, F. Rossi, Capability chart for electrical power systems, IEE Gen. Transm. Distrib., 139 (1992) 71-75.

[18] P. Cuffe, P. Smith, A. Keane, Characterisation of the reactive power capability of diverse distributed generators: Toward an optimisation approach, IEEE Power and Energy Soc. Gen. Meeting, 2012.

[19] P. E. Gill, W. Murray, M. H. Wright, Practical Optimization, Academic Press, 1981.

[20] M. Cho, Y. Chen, Fixed/switched type shunt capacitor planning of distribution systems by considering customer load patterns and simplified feeder model, IEE Gen. Transm. Distrib., 144 (1997) 533-540.

[21] IEEE 13 Node Test Feeder, [Online]. Available: http://ewh.ieee.org/soc/pes/dsacom/testfeeders/ index.html, 03 June 2013.

[22] P. Kundur, Power System Stability and Control, McGraw-Hill, 1994. 
[23] A. Masoum, S. Deilami, P. Moses, M. Masoum, A. Abu-Siada, Smart load management of plugin electric vehicles in distribution and residential networks with charging stations for peak shaving and loss minimisation considering voltage regulation, IEE Gen. Transm. Distrib., 5 (2011) 877-88.

[24] Z. Liu, F. Wen, G. Ledwich, Optimal planning of electric-vehicle charging stations in distribution systems, IEEE Trans. Power Del., 28 (2013) 102-110.

[25] M. Yilmaz, P. Krein, Review of the impact of vehicle-to-grid technologies on distribution systems and utility interfaces, IEEE Power Electronics, 28 (2013) 5673-5689.

[26] C. Guille, G. Gross, A conceptual framework for the vehicle-to-grid (V2G) implementation, Energy Policy, 37 (2009) 4379 - 4390. 